\title{
Extraction Of Hydrophobic Species Into A Water-Soluble Synthetic Receptor
}

\section{Supporting Information}

Richard J. Hooley, Hillary J. Van Anda and Julius Rebek, Jr.*

The Skaggs Institute for Chemical Biology and the Department of Chemistry, The Scripps

Research Institute MB-26, 10550 North Torrey Pines Rd., La Jolla, CA 92037.

jrebek@scripps.edu

\section{Selected NMR Experiments}
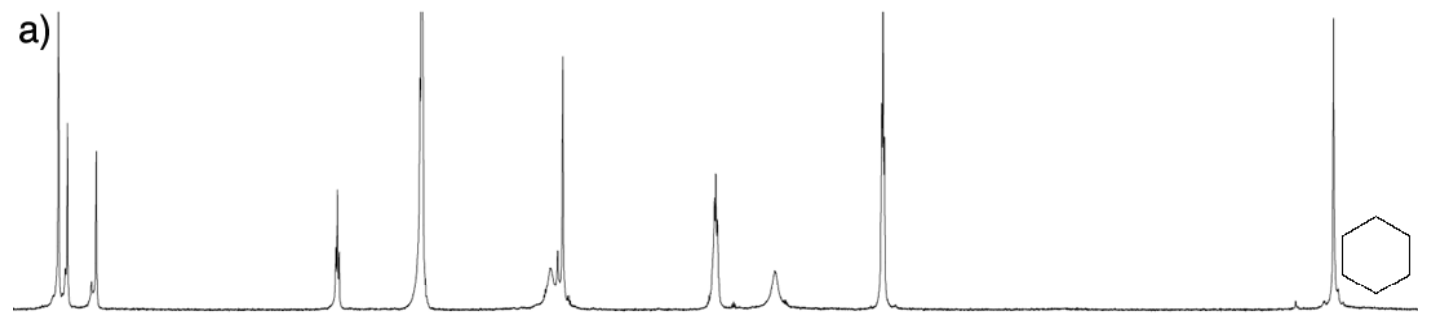

b)

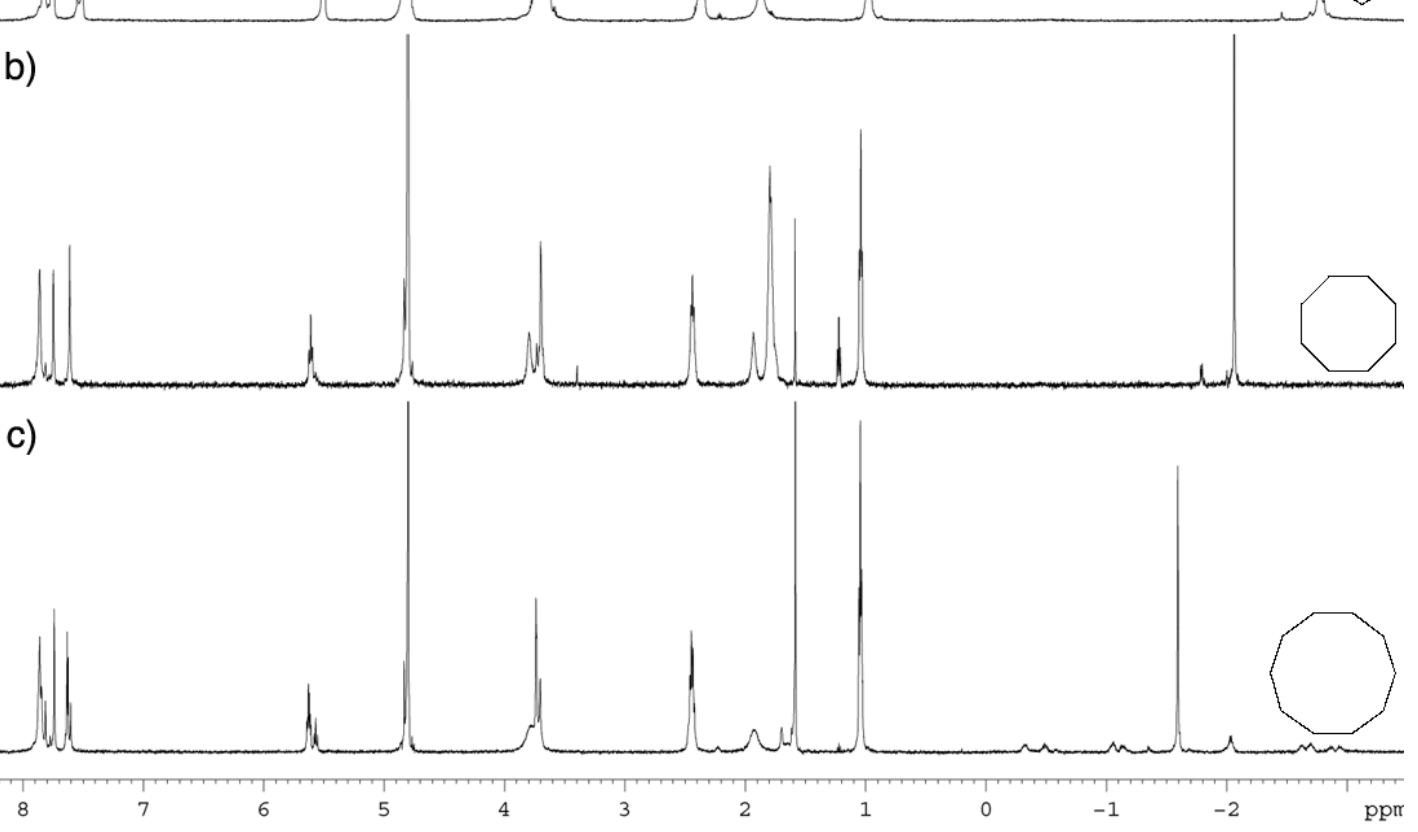

c)

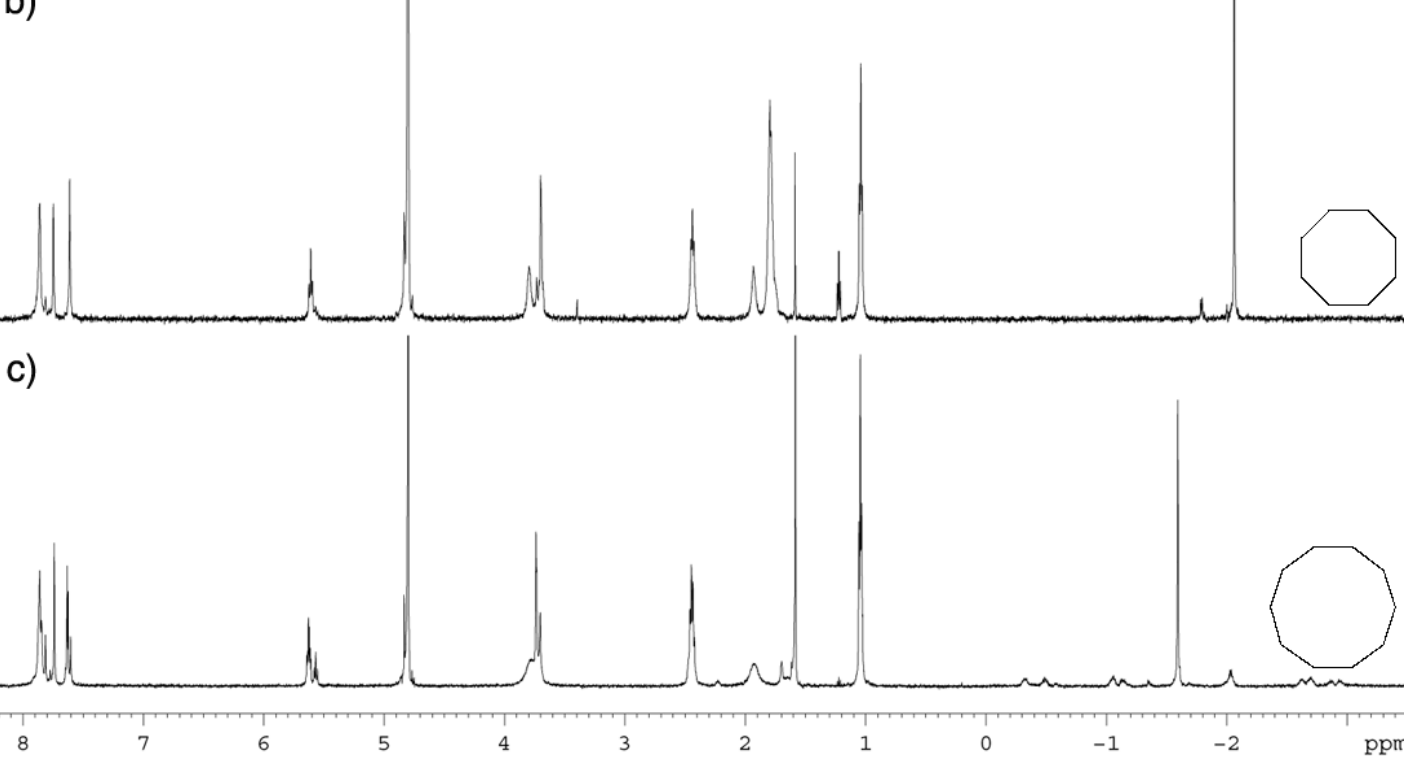

Figure $\boldsymbol{S}$-1. ${ }^{1} \mathrm{H}$ NMR spectra $(600 \mathrm{MHz})$ of $2 \mathrm{mM}$ cavitand 1 and cycloalkanes in $\mathrm{D}_{2} \mathrm{O}$ : (a) cyclohexane, (b) cyclooctane, (c) cyclodecane. 


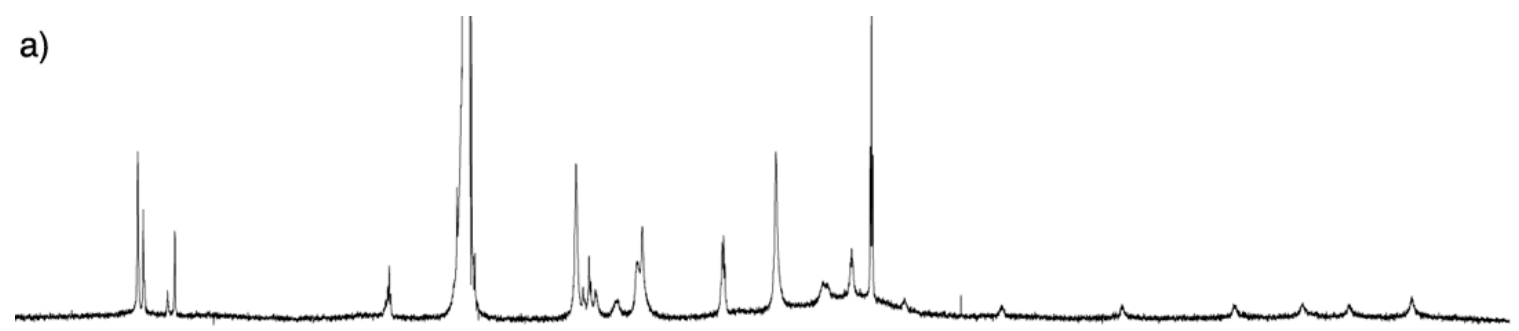

b)

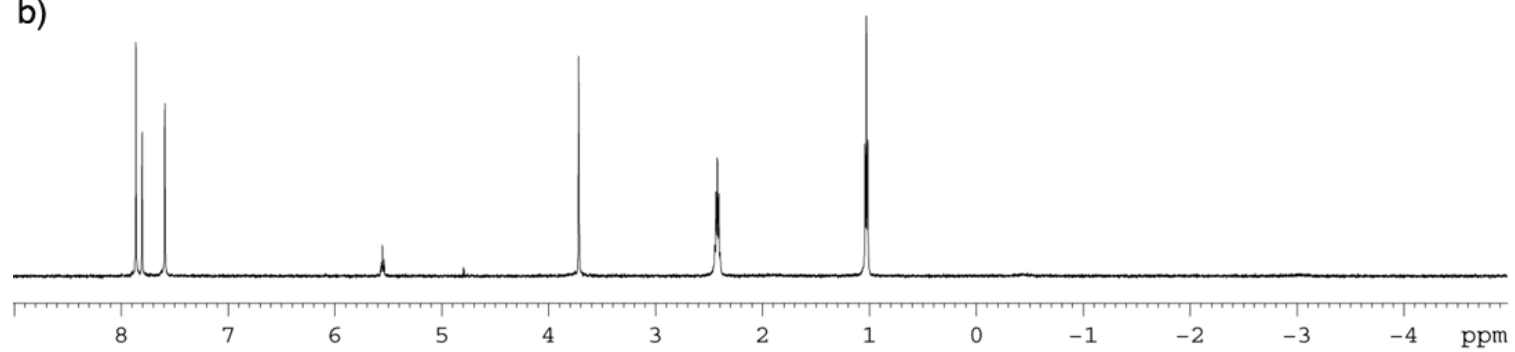

Figure S-2. ${ }^{1} \mathrm{H}$ NMR spectra (600 MHz) of (b) $1 \mathrm{mM}$ host 1 in $\mathrm{D}_{2} \mathrm{O}$, and (a) in the presence of one equivalent dodecyltrimethylammonium bromide - showing the alkyl chain bound within the cavity in a helical conformation.

Table $\boldsymbol{S}$-1. Observed and predicted (calculated) chemical shifts for $n$-alkanes complexed with cavitand 1 in $\mathrm{D}_{2} \mathrm{O}(2 \mathrm{mM}, 600 \mathrm{MHz})$.

\begin{tabular}{|c|c|c|c|}
\hline SDS observed $\delta$ 's & n-alkane & Predicted $\boldsymbol{\delta}$ 's & Observed $\delta$ 's \\
\hline & HEXANE & Predicted & Observed \\
\hline $\mathrm{C}_{1}:-3.95$ & $\mathrm{C}_{1 / 6}$ & -2.28 & -2.17 \\
\hline $\mathrm{C}_{2}:-3.35$ & $\mathrm{C}_{2 / 5}$ & -2.33 & -2.17 \\
\hline $\mathrm{C}_{3}:-2.95$ & $\mathrm{C}_{3 / 4}$ & -2.63 & -2.67 \\
\hline $\mathrm{C}_{4}:-2.30$ & & & \\
\hline $\mathrm{C}_{5}:-1.30$ & $\mathrm{HEPTANE}$ & Predicted & Observed \\
\hline $\mathrm{C}_{6}:-0.20$ & $\mathrm{C}_{1 / 7}$ & -1.83 & -1.80 \\
\hline $\mathrm{C}_{7}: 0.70$ & $\mathrm{C}_{2.6}$ & -1.78 & -1.80 \\
\hline $\mathrm{C}_{8}: 1.10$ & $\mathrm{C}_{3 / 5}$ & -2.13 & -2.10 \\
\hline $\mathrm{C}_{9}: 1.20$ & $\mathrm{C}_{4}$ & -2.40 & -2.37 \\
\hline $\mathrm{C}_{10}: 1.20$ & & & \\
\hline $\mathrm{C}_{11}: 1.20$ & $\mathrm{OCTANE}$ & Predicted & Observed \\
\hline & $\mathrm{C}_{1 / 8}$ & -1.63 & -1.59 \\
\hline & $\mathrm{C}_{2 / 7}$ & -1.33 & -1.35 \\
\hline & $\mathrm{C}_{3 / 6}$ & -1.58 & -1.59 \\
\hline & $\mathrm{C}_{4 / 5}$ & -1.80 & -1.83 \\
\hline & & & \\
\hline & NONANE & Predicted & Observed \\
\hline
\end{tabular}




\begin{tabular}{|c|c|c|c|}
\hline & $\mathrm{C}_{1 / 9}$ & -1.58 & -1.50 \\
\hline & $\mathrm{C}_{2 / 8}$ & -1.13 & -1.11 \\
\hline & $\mathrm{C}_{3 / 7}$ & -1.13 & -1.11 \\
\hline & $\mathrm{C}_{4 / 6}$ & -1.25 & -1.25 \\
\hline & $\mathrm{C}_{5}$ & -1.40 & -1.50 \\
\hline & & & \\
\hline & DECANE & Predicted & Observed \\
\hline & $\mathrm{C}_{1 / 10}$ & -1.58 & -1.48 \\
\hline & $\mathrm{C}_{2 / 9}$ & -1.08 & -1.00 \\
\hline & $\mathrm{C}_{3 / 8}$ & -0.93 & -0.91 \\
\hline & $\mathrm{C}_{4 / 7}$ & -0.80 & -0.82 \\
\hline & $\mathrm{C}_{5 / 6}$ & -0.75 & -0.75 \\
\hline & & & \\
\hline & $\mathrm{UNDCANE}$ & Predicted & Observed \\
\hline & $\mathrm{C}_{1 / 11}$ & -1.58 & -1.49 \\
\hline & $\mathrm{C}_{2 / 10}$ & -1.08 & -0.99 \\
\hline & $\mathrm{C}_{3 / 9}$ & -0.88 & -0.83 \\
\hline & $\mathrm{C}_{4 / 8}$ & -0.60 & -0.60 \\
\hline & $\mathrm{C}_{5 / 7}$ & -0.30 & -0.33 \\
\hline & $\mathrm{C}_{6}$ & -0.30 & -0.23 \\
\hline
\end{tabular}

Table 5. Concentration dependence of exchange rates between $1(2 \mathrm{mM})$ and bulk solvent $\left(\mathrm{D}_{2} \mathrm{O}\right)$.

\begin{tabular}{|c|c|c|c|}
\hline Guest & {$[$ Guest $] / \mathbf{m M}$} & & $\Delta G^{\ddagger} / \mathrm{kcal}^{\mathrm{mol}^{-1}}$ \\
\hline & 18 & 10.0 & 16.2 \\
\hline & 12 & 8.9 & 16.3 \\
\hline & 8 & 10.6 & 16.2 \\
\hline & 3 & 9.8 & 16.2 \\
\hline & 9 & 0.75 & 17.7 \\
\hline & 6 & 0.86 & 17.6 \\
\hline & 4.3 & 0.91 & 17.6 \\
\hline & 3.0 & 1.43 & 17.4 \\
\hline
\end{tabular}




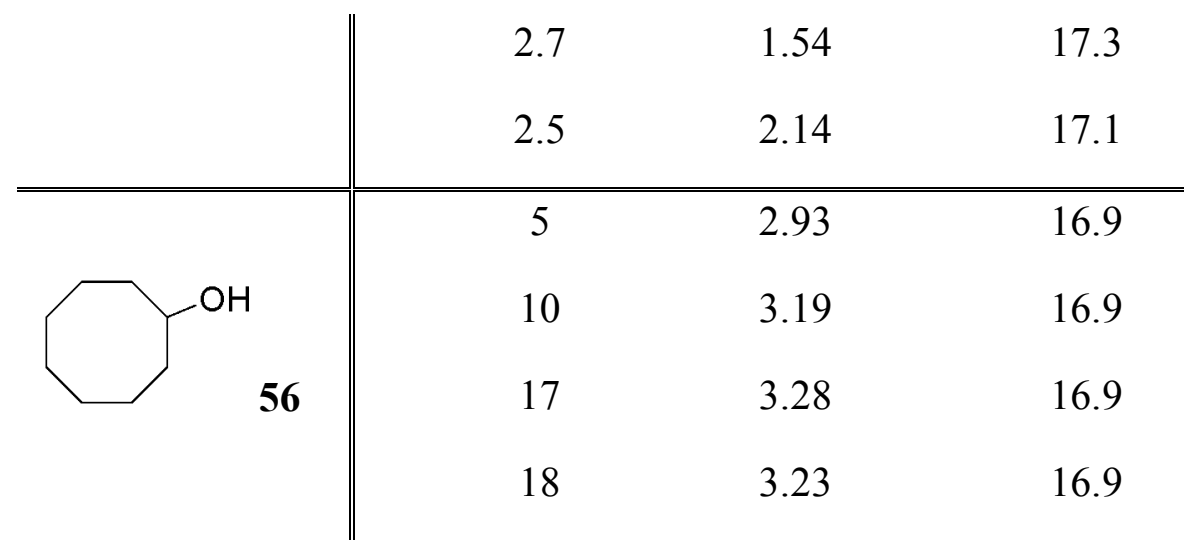

NOESY Spectra:

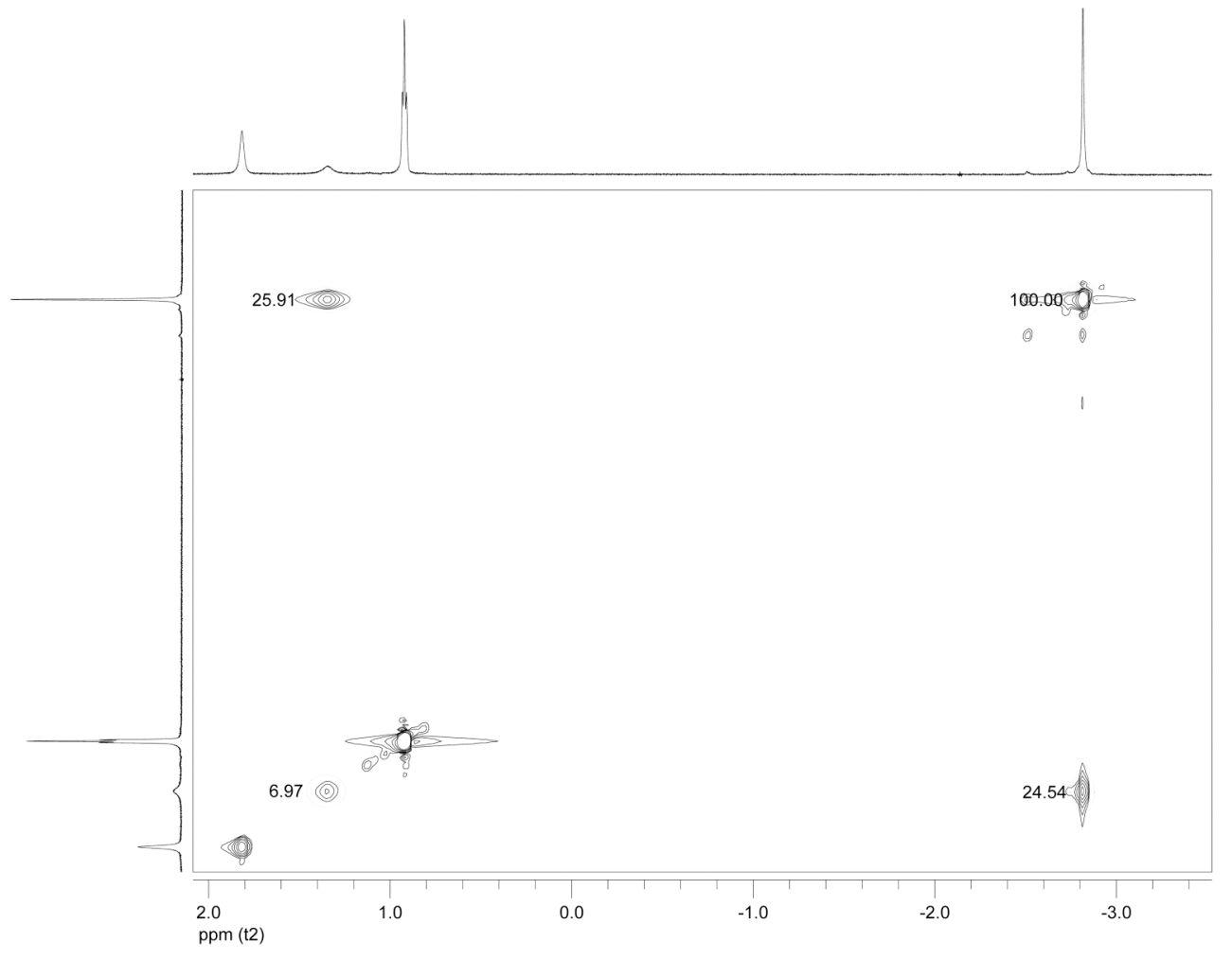

Figure $\boldsymbol{S} \mathbf{- 3}$. 2D NOESY spectrum of $\mathbf{1} \bullet$ cyclohexane, $300 \mathrm{~ms}$ mixing time. 


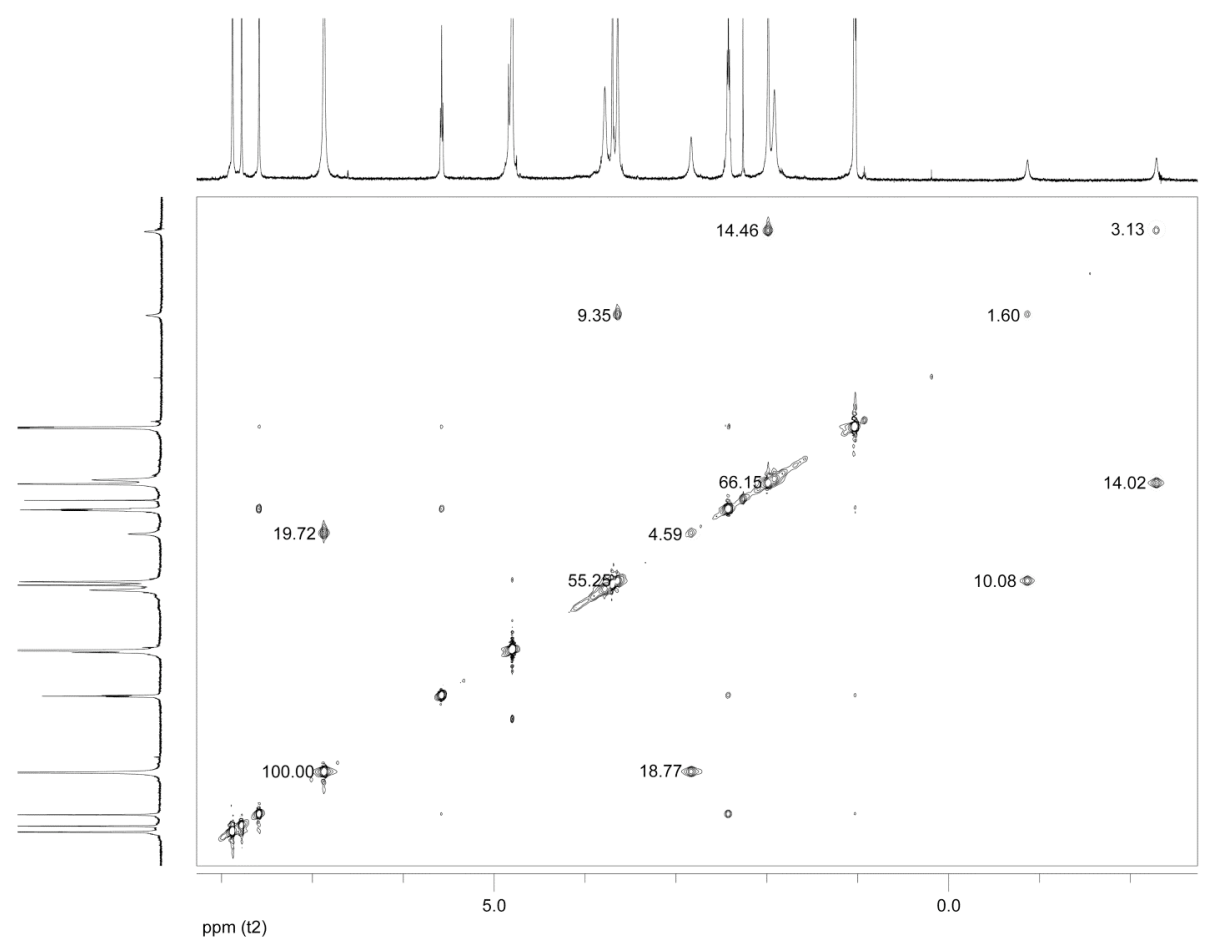

Figure $\boldsymbol{S}$-4. 2D NOESY spectrum of $\mathbf{1}$-norbornadiene, 300ms mixing time.

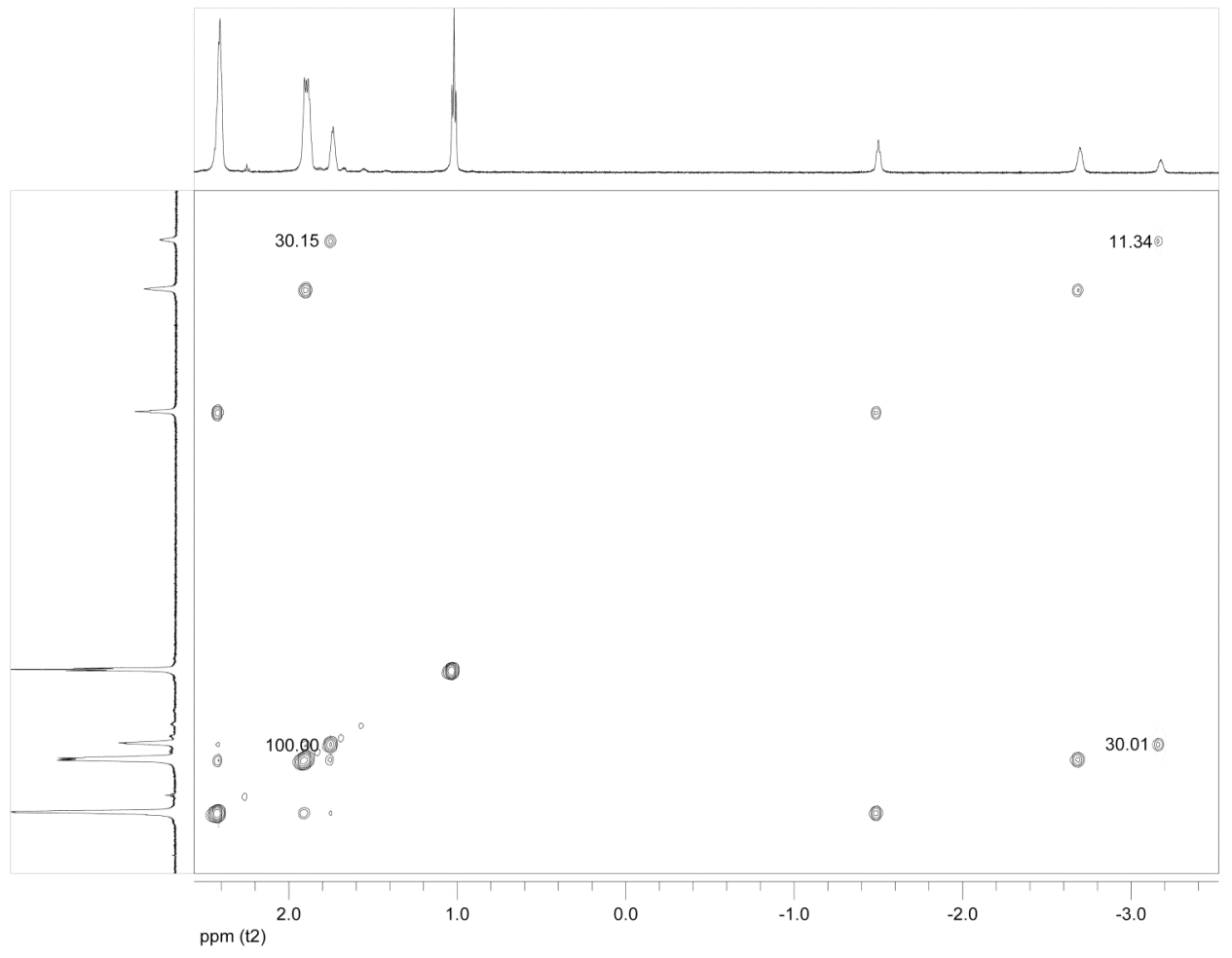

Figure $\boldsymbol{S}$-5. 2D NOESY spectrum of $\mathbf{1} \bullet$ cyclohexanone, $300 \mathrm{~ms}$ mixing time. 


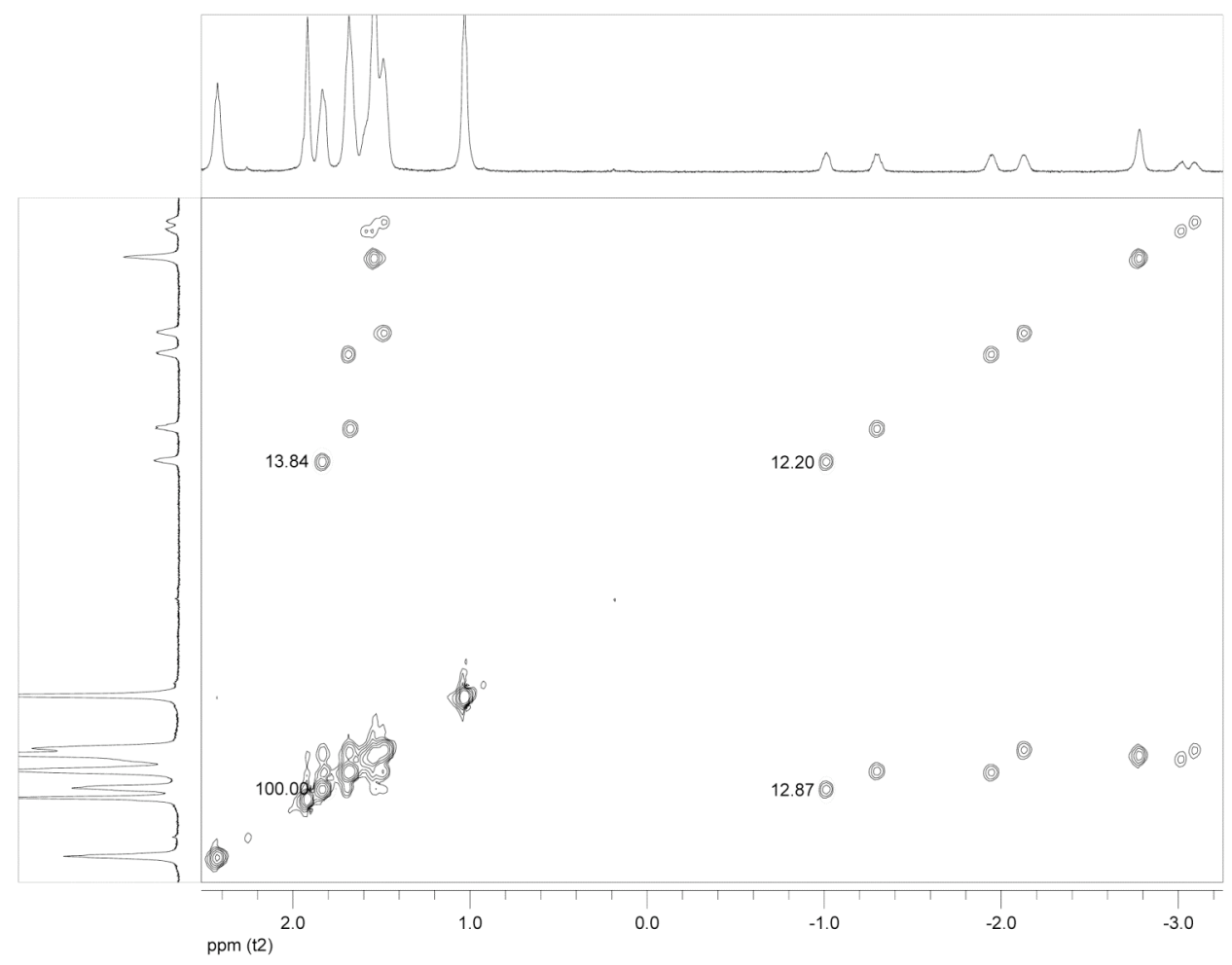

Figure $\boldsymbol{S}$-6. 2D NOESY spectrum of $\mathbf{1} \cdot$ cyclooctanol, 300ms mixing time.

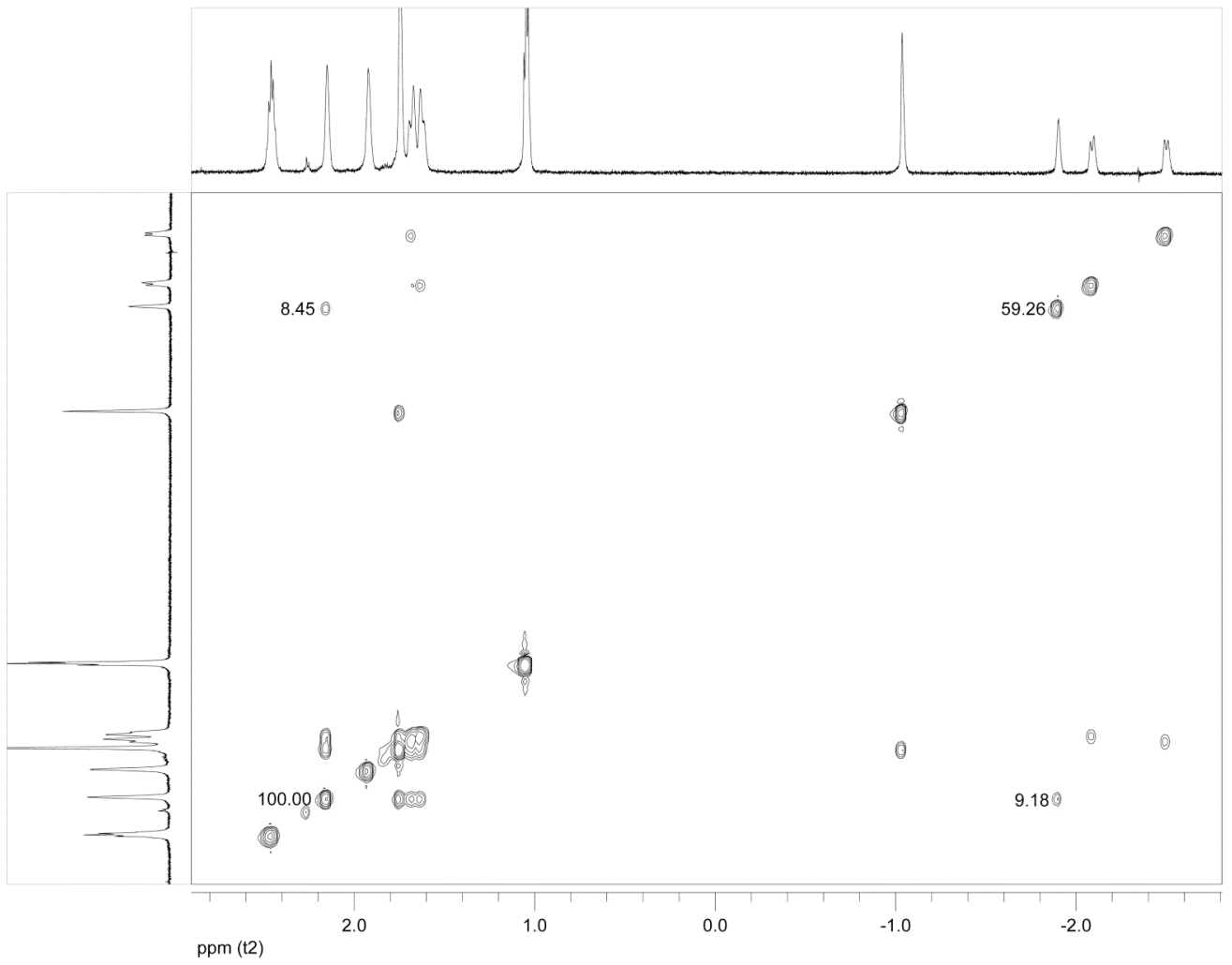

Figure $\boldsymbol{S}$-7. 2D NOESY spectrum of $\mathbf{1} \cdot 1$-adamantanol, 300ms mixing time. 


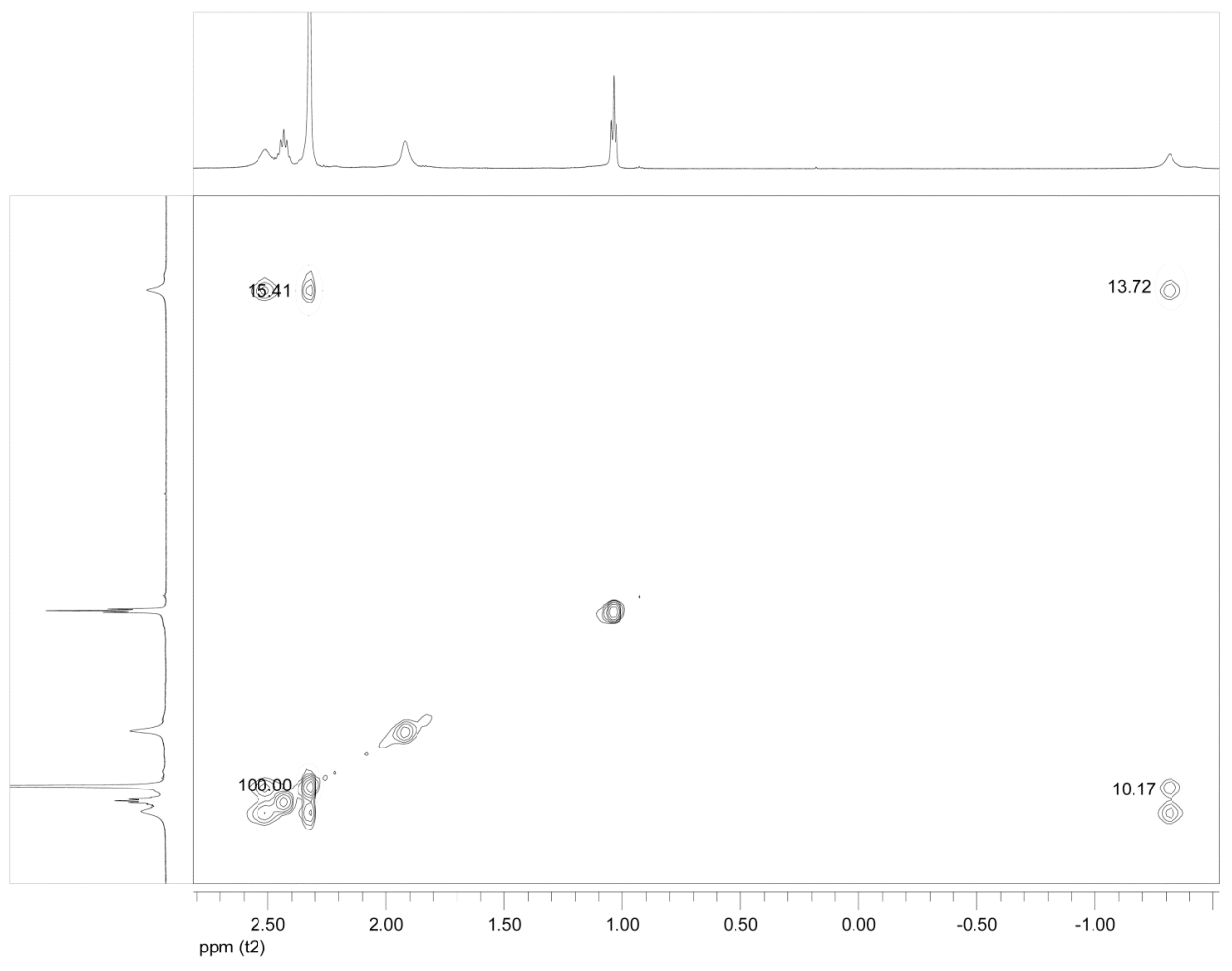

Figure $\boldsymbol{S}$-8. 2D NOESY spectrum of $\mathbf{1} \cdot 1,5$-cyclooctadiene, $300 \mathrm{~ms}$ mixing time. 


\section{Raw ITC Data}

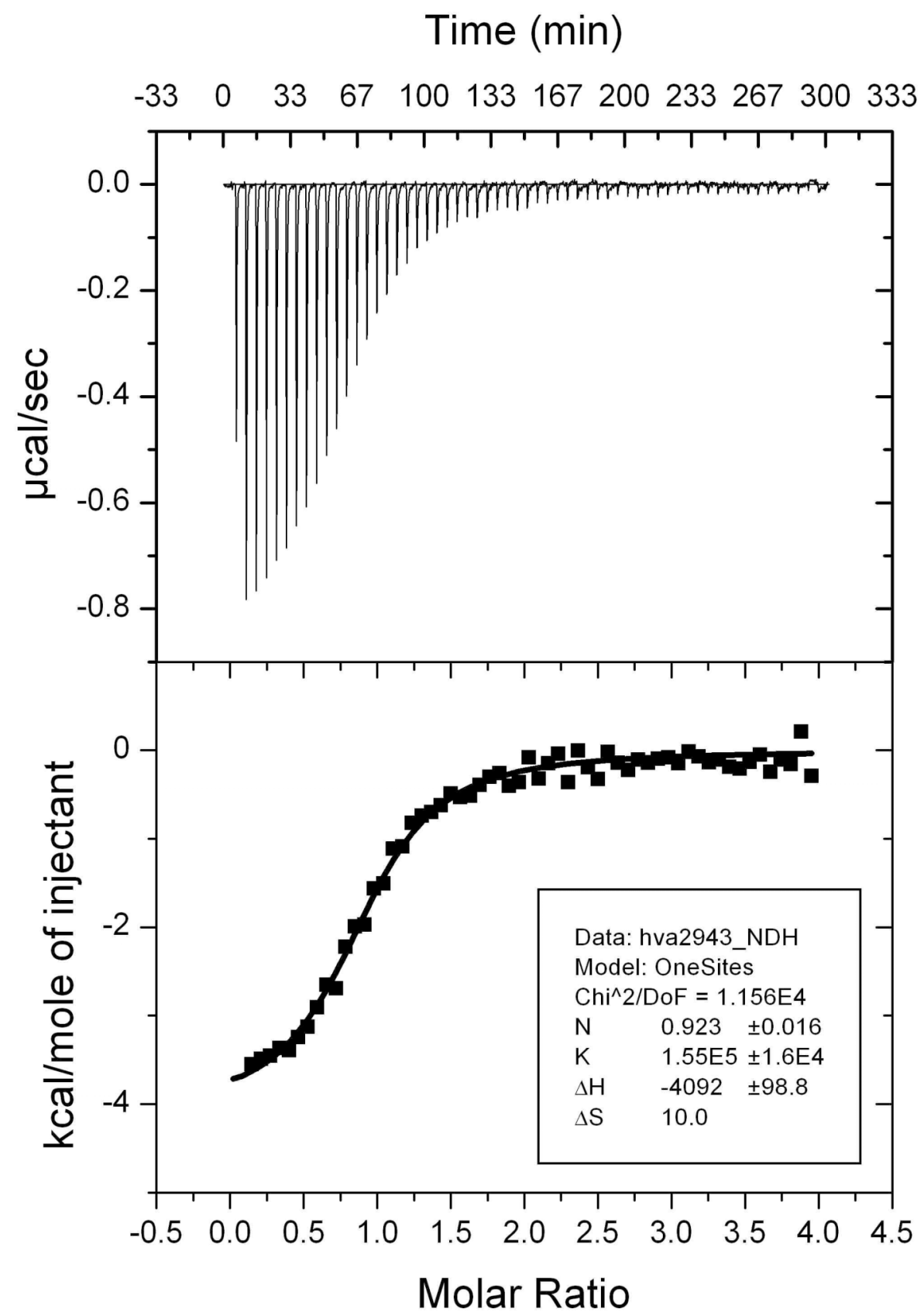

Figure $\boldsymbol{S}$-9. Cyclohexanone injected into $0.065 \mathrm{mM}$ cavitand 1 


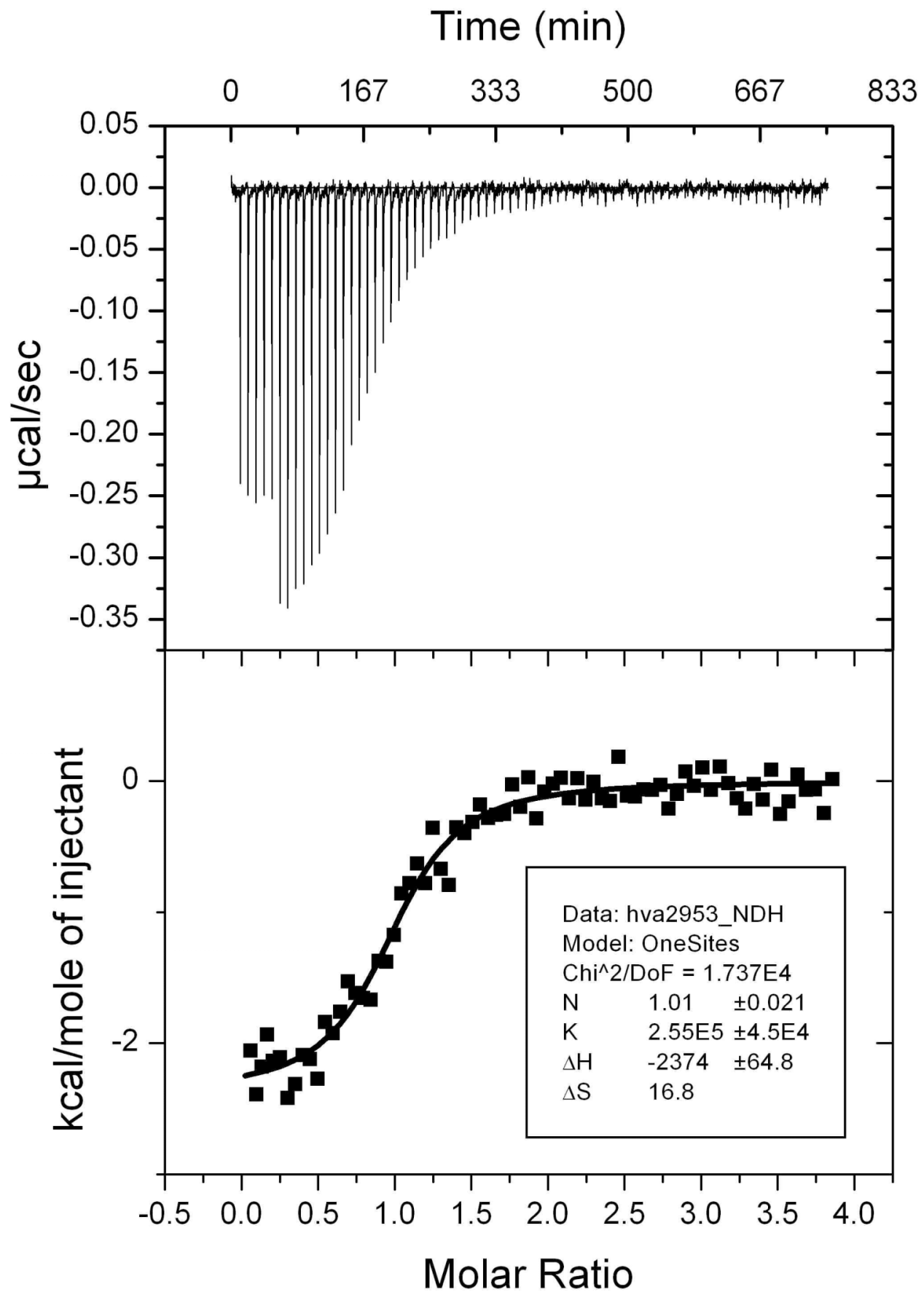

Figure $\boldsymbol{S}$-10. Cyclooctanol injected into $0.07 \mathrm{mM}$ cavitand $\mathbf{1}$ 


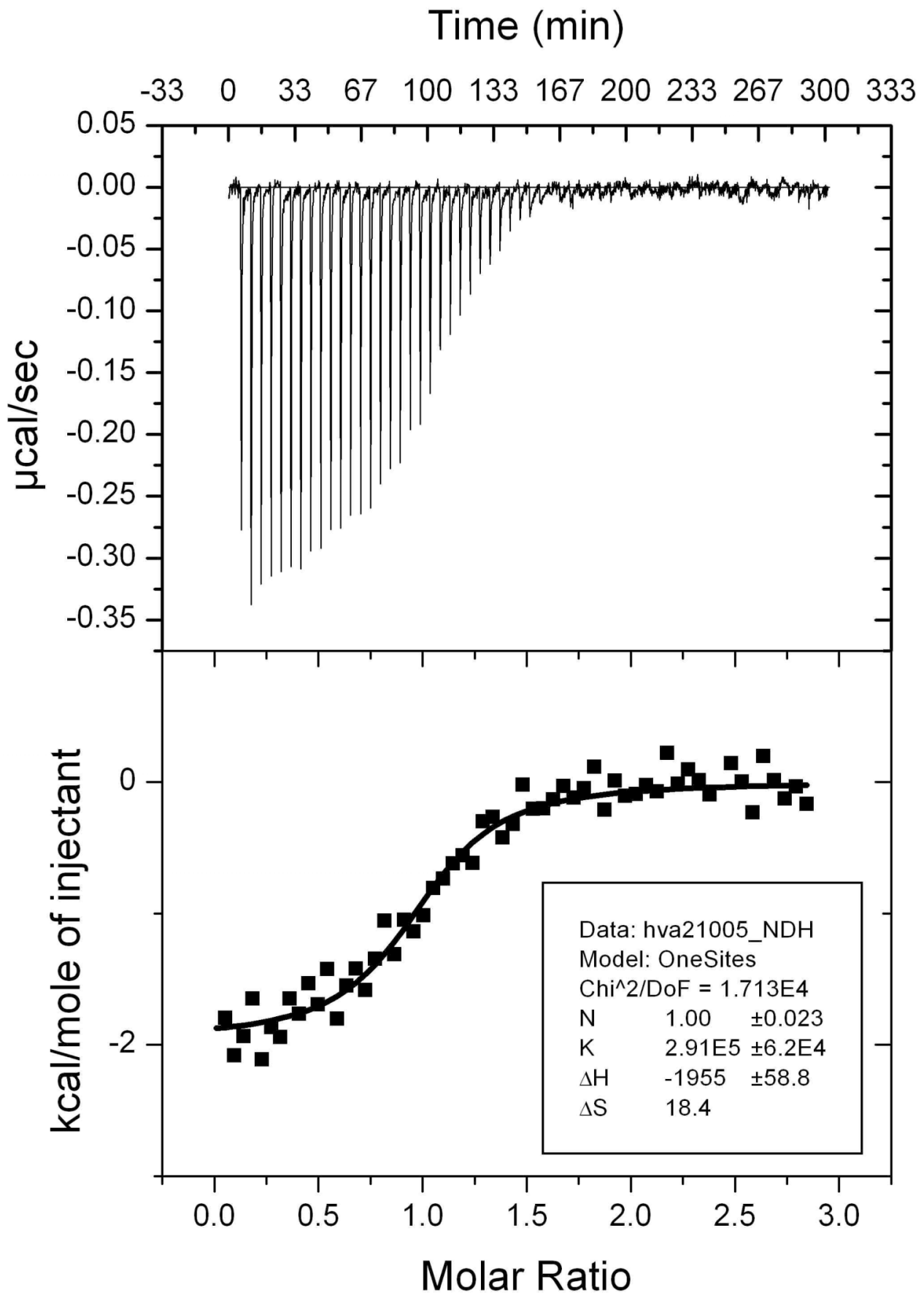

Figure $\boldsymbol{S}$-11. 1 -Adamantanemethanol injected into $0.08 \mathrm{mM}$ cavitand $\mathbf{1}$ 


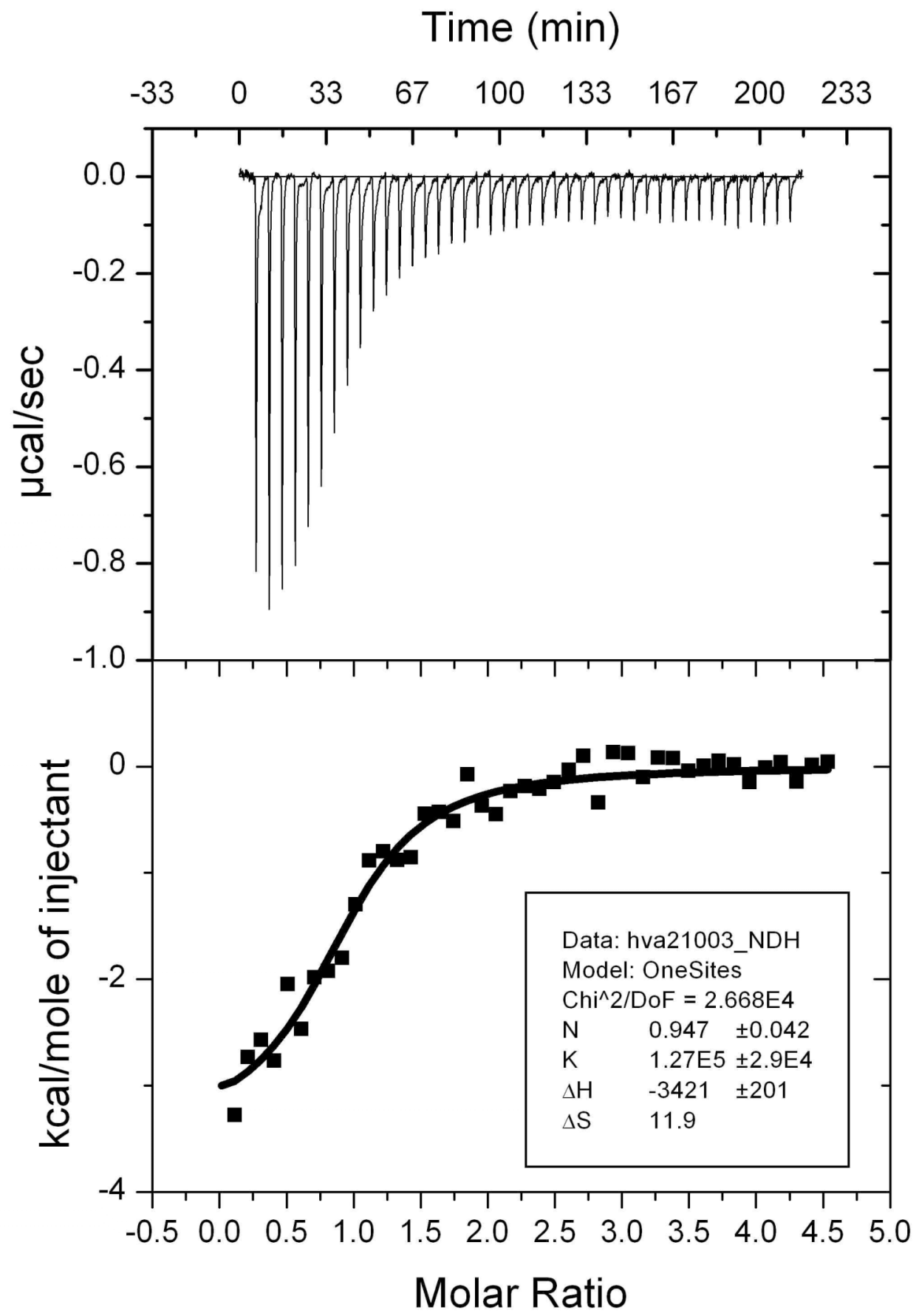

Figure $\boldsymbol{S}$-12. 2-Adamantanone injected into $0.06 \mathrm{mM}$ cavitand 1 


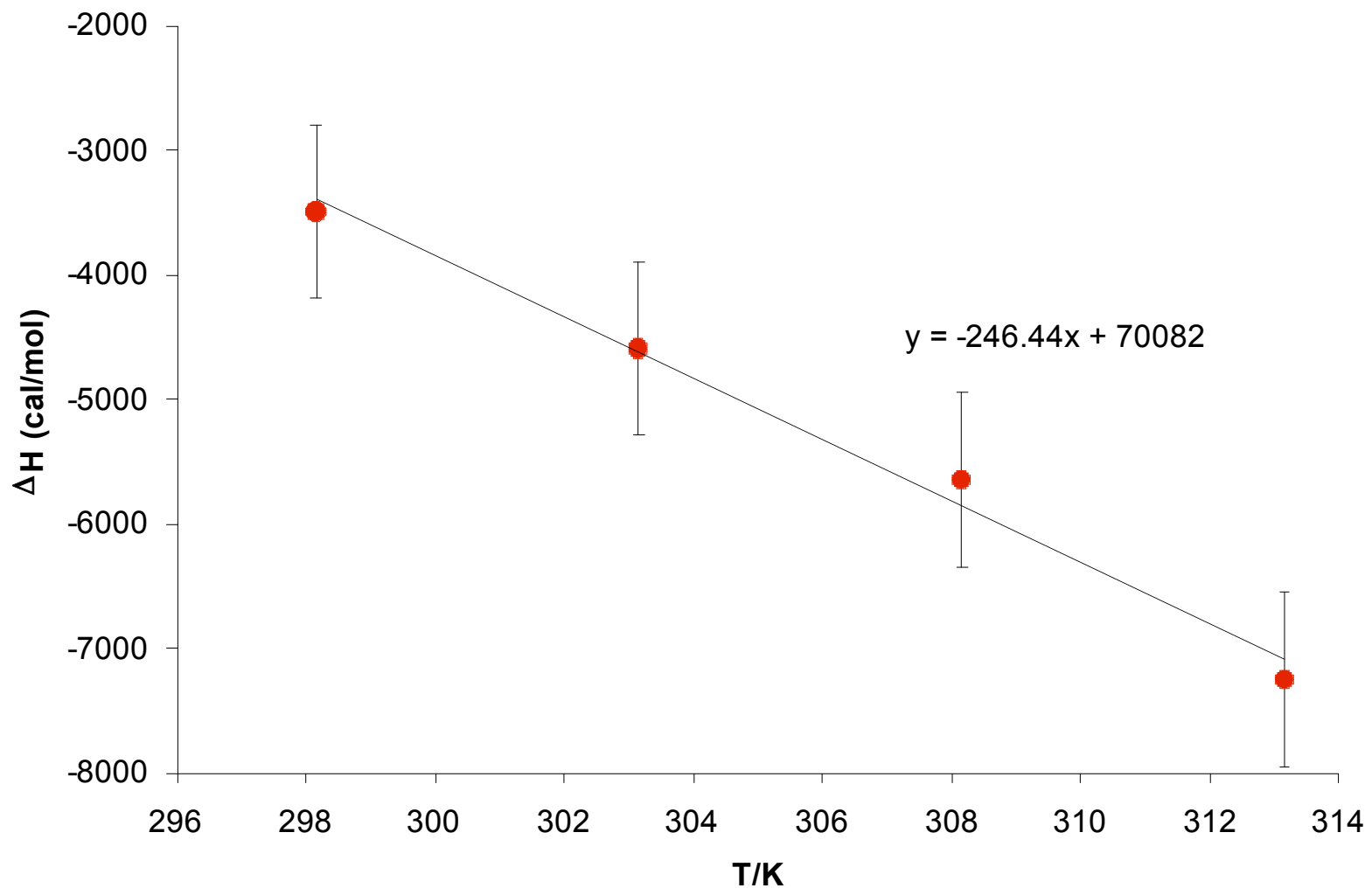

Figure $\boldsymbol{S}$-13. Temperature dependence of $\Delta \mathrm{H}_{\text {binding }}$ of cyclooctanol in cavitand $\mathbf{1}$ 